Abstract

\title{
Deep Mutational Scanning to Map How Zika Envelope Protein Mutations Affect Viral Growth and Antibody Escape ${ }^{\dagger}$
}

\author{
Marion Sourisseau 1, Daniel J. P. Lawrence 2,3, Megan C. Schwarz ${ }^{1}$, Carina H. Storrs 1, \\ Ethan C. Veit ${ }^{1}$, Jesse D. Bloom 2,3,4 and Matthew J. Evans 1,* \\ 1 Department of Microbiology, Icahn School of Medicine at Mount Sinai, New York, NY 10029, USA; \\ msouriss@gmail.com (M.S.); megan.schwarz@mssm.edu (M.C.S.); storrsc@gmail.com (C.H.S.); \\ ethan.veit@icahn.mssm.edu (E.C.V.) \\ 2 Basic Sciences Division and Computational Biology Program, Fred Hutchinson Cancer Research Center, \\ Seattle, WA 98109, USA; djpl@uw.edu (D.J.P.L.); jbloom@fredhutch.org (J.D.B.) \\ 3 Department of Genome Sciences, University of Washington, Seattle, WA 98195, USA \\ 4 Howard Hughes Medical Institute, Seattle, WA 98109, USA \\ * Correspondence: matthew.evans@mssm.edu \\ † Presented at Viruses 2020-Novel Concepts in Virology, 5-7 February 2020. \\ Published: 23 June 2020
}

\begin{abstract}
The Zika virus has recently been shown to be associated with severe birth defects. The virus' envelope (E) protein mediates its ability to infect cells and is also the primary target of the antibodies that are elicited by natural infection and the vaccines that are being developed against the virus. Therefore, determining the effects of mutations on this protein is important for understanding its function, its susceptibility to vaccine-mediated immunity, and its potential for future evolution. Functional constraints on viral proteins are often assessed by examining sequence conservation among natural strains, but this approach is relatively ineffective for the Zika virus because all known sequences are highly similar. Here, we take an alternative approach to mapping functional constraints on Zika virus' E protein by using deep mutational scanning to measure how all amino-acid mutations in the protein affect viral growth in cell culture. The resulting sequencefunction map is consistent with the existing knowledge about $\mathrm{E}$ protein structure and function but also provides insight into mutation-level constraints in many regions of the protein that have not been well characterized in prior functional work. In addition, we extend our approach to completely map how mutations affect viral neutralization by two monoclonal antibodies, thereby precisely defining their functional epitopes. Overall, our study provides a valuable resource for understanding the effects of mutations on this important viral protein and also offers a roadmap for future work to map functional and antigenic selection to the Zika virus at high resolution.
\end{abstract}

Keywords: deep mutagenesis; evolution; diversity: immune epitopes

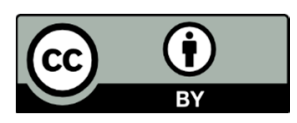

(C) 2020 by the authors. Licensee MDPI, Basel, Switzerland. This article is an open access article distributed under the terms and conditions of the Creative Commons Attribution (CC BY) license (http://creativecommons.org/licenses/by/4.0/). 